Marisa Biesdorf, Jean Carlos de Souza,

Luana da Cruz Santos, Cybelle D. Soares Schlemper e

Clair Antônio Wathier

\title{
O CONTEXTO DO AMBIENTE PERCEBIDO NA ATIVIDADE FÍSICA DE LAZER E DESLOCAMENTO EM IDOSOS
}

Recebido em: $12 / 06 / 2017$

Aceito em: 27/03/2018

Giovane Pereira Balbé $^{1}$
Marisa Biesdorf
Jean Carlos de Souza
Luana da Cruz Santos
${ }^{3}$
Cybelle Doederlein Soares Schlemper
Clair Antônio Wathier
Centro Universitário para o Desenvolvimento do Alto Vale do Itajaí (UNIDAVI)
Rio do Sul - SC - Brasil

RESUMO: O objetivo deste estudo é comparar a prevalência de Atividade Física (AF) no lazer e deslocamento com a percepção do ambiente em idosos. Estudo transversal, conduzido com 213 idosos do município de Rio do Sul - SC. A AF no lazer e deslocamento foram investigadas conforme os domínios do Questionário Internacional de Atividade Física. A percepção do ambiente foi avaliada mediante a Neighborhood Environmental Walkability Scale. O deslocamento apresentou diferença significativa entre os idosos que relataram maior densidade residencial próxima de sua residência, a existência de lugares para caminhar e andar de bicicleta, melhor estética do bairro e maior segurança no trânsito. Não houve relação na AF de lazer e os domínios do ambiente. Conclui-se que a AF no deslocamento em idosos pode ser mais suscetível a influência das características ambientais, em relação ao lazer.

PALAVRAS CHAVE: Atividade Motora. Meio Ambiente. Idoso.

\section{THE CONTEXT OF THE ENVIRONMENT PERCEIVED IN THE PHYSICAL ACTIVITY OF LEISURE AND COMMUTING IN OLDER ADULTS}

ABSTRACT: The objective of this study is to compare the prevalence of Physical Activity (AF) in leisure and communing with the perception of the environment in the

\footnotetext{
1 Mestre em Ciências do Movimento Humano. Professor no curso de Educação Física do Centro Universitário para o Desenvolvimento do Alto Vale do Itajaí - UNIDAVI.

${ }^{2}$ Bacharel em Educação Física pelo Centro Universitário para o Desenvolvimento do Alto Vale do Itajaí UNIDAVI

${ }^{3}$ Bacharel em Educação Física pelo Centro Universitário para o Desenvolvimento do Alto Vale do Itajaí UNIDAVI

${ }^{4}$ Bacharel em Educação Física pelo Centro Universitário para o Desenvolvimento do Alto Vale do Itajaí UNIDAVI

${ }^{5}$ Mestre em Educação Física pela Universidade Federal de Santa Catarina - UFSC

${ }^{6}$ Especialista em Fisiologia do Exercício. Professor no Centro Universitário para o Desenvolvimento do Alto Vale do Itajaí - UNIDAVI
} 
Marisa Biesdorf, Jean Carlos de Souza,

Luana da Cruz Santos, Cybelle D. Soares Schlemper e

Clair Antônio Wathier

older adults. Cross sectional study, conducted with 213 older adults people from the city of Rio do Sul - SC. The AF in the leisure and communing were investigated according to the domains of the International Questionnaire of Physical Activity. The perception of the environment was evaluated through the Neighborhood Environmental Walkability Scale. The communing showed a significant difference between the older adults who reported higher residential density near their residence, the existence of places for walking and cycling, better aesthetics of the neighborhood and greater safety in traffic. There was no relation in leisure AF and the domains of the environment. It can be concluded that the AF in the communing in the older adults can be more susceptible to the influence of the environmental characteristics, in relation to the leisure.

KEYWORDS: Motor Activity. Environment. Aged.

\section{Introdução}

Diretrizes científicas emitidas por diversos órgãos e centros de pesquisa internacionais têm documentado que a atividade física regular apresenta-se como fator de proteção contra doenças coronárias, diabetes tipo dois, alguns tipos de câncer, hipertensão, obesidade, depressão clínica, entre outras doenças crônicas (NELSON et al., 2007; WHO, 2010).

Apesar do conhecimento quanto aos benefícios oriundos dessa atividade, ainda é baixa a proporção de indivíduos ativos, especialmente na população idosa (HENSON et al., 2013). Estudo (DUMITH et al., 2011) multicêntrico realizado para investigar a prevalência de inatividade física na população acima de 15 anos de idade, conduzido em 76 países, constatou que a inatividade física foi mais prevalente entre os países mais desenvolvidos, especialmente entre as mulheres e os idosos.

Nesse sentido, Heath et al. (2012) ao revisarem estudos de intervenção de atividade física realizados no mundo, publicados entre 2000 e 2011, identificaram que as abordagens comportamentais e sociais são efetivas para o aumento da prática de atividade física. Além disso, abordagens ambientais e políticas de planejamento que incluem a criação e melhoria do acesso aos locais de atividade física, estruturação 
Marisa Biesdorf, Jean Carlos de Souza,

Luana da Cruz Santos, Cybelle D. Soares Schlemper e

Clair Antônio Wathier

urbana e práticas de transporte ativo permitem aumentar os níveis de atividade física entre as pessoas de várias idades e de diferentes grupos sociais.

A partir de 1990 aumentou o interesse dos pesquisadores pelo estudo em ambientes (natural, construído e social), especialmente a nível internacional, apontandoo como fator de influência na atividade física em idosos (BENGOECHEA; SPENCE; McGANNON, 2005; HOEHNER et al., 2005; LIBRETT; YORE; SCHMID, 2006), pois as limitações estruturais no ambiente representam obstáculos para a prática de atividade física (PIZANI et al., 2015), já que os idosos que não residem próximos a instalações e espaços de lazer tendem a ser menos ativos fisicamente (BOOTH et al., 2000; GALLAGHER et al., 2010).

No Brasil, a pesquisa sobre percepção do ambiente e prática de atividade física em idosos ainda é recente. Os primeiros estudos foram conduzidos com idosos residentes em Ermelino Matarazzo, zona leste do município de São Paulo (SALVADOR et al., 2009; SALVADOR; REIS; FLORINDO, 2009), seguido de um estudo conduzido em Florianópolis (GIEHL et al., 2012) e mais recentemente em Rio do Sul (BALBÉ; WATHIER; RECH, 2017).

Cabe destacar que esses estudos foram direcionados a percepção do ambiente e a prática de atividade física no lazer ou deslocamento de forma geral e com idosos residentes em grandes centros urbanos. Além disso, o estudo recente (BALBÉ; WATHIER; RECH, 2017) envolve apenas a caminhada como forma de lazer e deslocamento, não fornecendo uma visão geral desses domínios da atividade física, considerando o contexto de pequenos centros urbanos. Nesse sentido, Dumith et al. (2011) ressaltam que embora os dados disponíveis para a atividade física sejam na maioria relacionada ao lazer, os mesmos apresentam informações imprecisas. 
Com isso, este estudo tem como objetivo identificar e comparar a prevalência da prática de atividade física no deslocamento e no lazer com a percepção do ambiente em idosos residentes em Rio do Sul, Santa Catarina.

\section{Método}

Estudo de natureza descritiva e transversal conduzido em 2014 no município de Rio do Sul, Santa Catarina.

O município de Rio do Sul está localizado no Alto Vale do Itajaí, em Santa Catarina, com uma área de 260,357 $\mathrm{Km}^{2}$ e população aproximada de 61.198 habitantes, sendo 6.090 pertencentes a faixa etária com idade igual ou superior a 60 anos residentes na zona urbana (IBGE, 2010). Além disso, apresenta um Índice de Desenvolvimento Humano (IDH) municipal de 0,802 , o que o coloca em $36^{\text {a }}$ posição entre os municípios brasileiros com maior IDH (PNUD, 2013).

A amostra foi constituída por 213 idosos residentes na zona urbana de Rio do Sul. O processo de amostragem foi não probabilístico por acessibilidade. A fim de obter um número maior de idosos e de diferentes ambientes, optou-se por entrevistas na própria residência do idoso ou em locais públicos como, associações comunitárias, grupos de convivência, praças e postos de saúde. Desse modo, participaram do estudo indivíduos com 60 anos ou mais de idade, residentes na zona urbana de Rio do Sul, considerados independentes para atividades do cotidiano e com condições físicas e mentais para responder sozinho a entrevista. Esses aspectos foram avaliados no primeiro contato com o idoso, mediante observação.

A coleta de dados foi conduzida por entrevistadores formados e acadêmicos em educação física, previamente treinados. O treinamento consistiu na familiarização com 
Marisa Biesdorf, Jean Carlos de Souza,

Luana da Cruz Santos, Cybelle D. Soares Schlemper e

Clair Antônio Wathier

os questionários e a homogeneização das condutas ao aplicar a entrevista. Os idosos foram informados sobre os objetivos do estudo e foi solicitada a assinatura do Termo de Consentimento Livre e Esclarecido, atendendo aos preceitos éticos vigentes.

Este projeto foi aprovado no Comitê de Ética em Pesquisa (CEP) da Universidade do Vale do Itajaí, conforme parecer $n^{\circ} 685.806$ de 18 de junho de 2014.

Para a avaliação das características sociodemográficas foi utilizado um questionário elaborado pelo grupo de pesquisadores do estudo, com o objetivo de identificar informações quanto a: idade, anos de estudo, estado civil, renda per capta, ocupação, cor da pele e com quem mora.

A avaliação da prática de atividade física no deslocamento e no lazer ocorreu com a aplicação do Questionário Internacional de Atividade Física (IPAQ), versão longa, adaptado e validado para idosos no Brasil (BENEDETTI; MAZO; BARROS, 2004). O domínio do deslocamento inclui atividades realizadas como forma de deslocamento de um lugar para outro por meio da caminhada e uso de bicicleta. Já no lazer foi utilizado as questões referentes às atividades de recreação, esporte e exercício físico realizadas no tempo livre.

Para avaliação da percepção do ambiente foi utilizado a Neighborhood Environmental Walkability Scale (NEWS) traduzida e validada para a realidade brasileira (MALAVASI et al., 2007). A versão utilizada, composta de 74 questões, divididas em oito domínios: 1) densidade residencial (6 questões); 2) proximidades de lojas e comércio (31 questões); 3) acesso a serviços (6 questões); 4) conectividade das ruas (3 questões); 5) lugares para caminhar e andar de bicicleta (3 questões); 6) estética do bairro (4 questões); 7) segurança no trânsito (10 questões) e 8) criminalidade no bairro (11 questões). A primeira parte da escala é estruturada para que os idosos 
respondam quanto ao número de residências próximas de sua casa. Posteriormente, a escala avalia o tempo de caminhada da residência do avaliado em relação a existência ou não de lojas e comércio, seguido da facilidade de acesso a serviços e possibilidade de rotas alternativas para tal. Avalia também, a existência de locais para caminhar e andar de bicicleta, investigando a estética do bairro, a segurança no trânsito e no bairro. Os idosos foram orientados para considerar como locais próximos de sua residência àqueles que pudessem chegar caminhando em até 15 minutos.

Para cada domínio da percepção do ambiente foi calculado um escore conforme propõem Saelens et al. (2002). A partir da não normalidade dos dados segundo o teste de normalidade de Kolmogorov Smirnof, adotou-se o teste Kruskal- Wallis, com nível de significância de 5\%, na comparação da percepção do ambiente e a prática de atividade física no deslocamento e no lazer. Estas variáveis foram tratadas em três níveis: inativo (idosos que não atingiram o mínimo de 10 minutos contínuos de atividade física por semana), insuficientemente ativo (que realizaram entre 10 a 149 minutos por semana de atividade física) e fisicamente ativo (que cumpriram 150 minutos ou mais de atividade física por semana).

Os dados foram armazenados em um banco de dados no programa Excel e cada entrevistado foi cadastrado segundo um número codificador. A análise dos dados ocorreu mediante o pacote estatístico Statistical Package for Social Science (SPSS), versão 17.0.

\section{Resultados}

A maioria da amostra foi composta de mulheres (78,9\%), na faixa etária entre 60 a 69 anos de idade (52,6\%). Mais da metade dos idosos eram casados $(55,0 \%)$, com 
Giovane Pereira Balbé, O Contexto do Ambiente Percebido na Atividade Física de Lazer...

Marisa Biesdorf, Jean Carlos de Souza,

Luana da Cruz Santos, Cybelle D. Soares Schlemper e

Clair Antônio Wathier

escolaridade até 4 anos de estudo $(56,8 \%)$ e com renda per capta predominante entre 1 a

2 salários mínimos $(53,1 \%)$. Quanto às demais características sociodemográficas, a

maioria referiu cor da pele branca $(95,0 \%)$, sendo aposentado $(74,6 \%)$ e que residia com

o cônjuge (45,5\%), conforme (TABELA 1).

Tabela 1. Características sociodemográficas da amostra estudada. Rio do Sul, SC, 2014.

\begin{tabular}{|c|c|c|}
\hline Variáveis Sociodemográficas & $\mathrm{f}$ & $\% *$ \\
\hline \multicolumn{3}{|l|}{ ( } \\
\hline Masculino & 45 & 21,1 \\
\hline Feminino & 168 & 78,9 \\
\hline \multicolumn{3}{|l|}{ Faixa etária (anos) } \\
\hline 60 a 69 & 112 & 52,6 \\
\hline 70 a 79 & 82 & 38,5 \\
\hline 80 ou mais & 19 & 8,9 \\
\hline \multicolumn{3}{|l|}{ Estado Civil } \\
\hline Solteiro & 22 & 10,3 \\
\hline Casado & 116 & 54,5 \\
\hline Viúvo/Separado/Divorciado & 75 & 35,2 \\
\hline \multicolumn{3}{|l|}{ Anos de estudo } \\
\hline 0 a 4 & 121 & 56,8 \\
\hline 5 a 8 & 55 & 25,8 \\
\hline 9 a 11 & 5 & 2,3 \\
\hline 12 ou mais & 32 & 15 \\
\hline \multicolumn{3}{|l|}{ Renda per capta (salário) ${ }^{\$}$} \\
\hline Menor que 1 & 16 & 7,5 \\
\hline 1 a 2 & 113 & 53,1 \\
\hline 3 a 4 & 60 & 28,2 \\
\hline 5 ou mais & 24 & 11,3 \\
\hline \multicolumn{3}{|l|}{ Cor da pele } \\
\hline Não Branca & 10 & 4,7 \\
\hline Branca & 203 & 95,3 \\
\hline \multicolumn{3}{|l|}{ Ocupação } \\
\hline Aposentado & 159 & 74,6 \\
\hline Aposentado e ativo/Remunerado ativo & 30 & 14,1 \\
\hline Não remunerado/Do lar & 24 & 11,3 \\
\hline \multicolumn{3}{|l|}{ Com quem Mora } \\
\hline Sozinho & 47 & 22,1 \\
\hline Cônjuge & 97 & 45,5 \\
\hline Com familiares/Outros & 69 & 32,4 \\
\hline Total & 213 & 100 \\
\hline
\end{tabular}

f = frequência; \%* = percentual ponderado; ${ }^{\$}$ Salário mínimo na época $R$ \$ 724,00.

$\mathrm{Na}$ Tabela 2, encontra-se a prevalência de idosos fisicamente ativos (150 minutos ou mais de atividade física por semana) que foi maior no lazer se comparada ao deslocamento (41,8\% e 30\%, respectivamente). A média de atividade física no lazer foi 
Marisa Biesdorf, Jean Carlos de Souza,

Luana da Cruz Santos, Cybelle D. Soares Schlemper e

Clair Antônio Wathier

de 166,5 minutos $(\mathrm{DP}=189,3$ minutos $)$ e no deslocamento foi de 123,3 minutos $(\mathrm{DP}=$ 163,1 minutos).

Essa maior prevalência de atividade física no lazer, aproximadamente $42 \%$ dos idosos entrevistados, é superior ao encontrado por Giehl et al. (2012) em Florianópolis (30\%). Nota-se que a atividade física no lazer é predominante entre a maioria dos idosos do município de Rio do Sul. Haja vista, se comparado ao deslocamento, a atividade física no lazer supera em quase $12 \%$. Acredita-se que tal resultado seja reflexo da origem étnica dos entrevistados, já que a maioria é europeia (alemão ou italiano) com uma cultura de lazer que envolve jogos como bocha, bolão, dança ou atividades festivas, diferentemente do cotidiano de idosos residentes num centro urbano maior e colonizado por açorianos como é o caso de Florianópolis.

Além disso, constatou-se que aproximadamente $75 \%$ dos idosos declararam-se aposentados enquanto apenas $14,1 \%$ declararam ainda trabalhar. Isso pode explicar em parte tais resultados, pois o idoso que não possui a necessidade diária de deslocar-se até o trabalho pode realizar menos atividade física de deslocamento por conta de sua condição. Por outro lado, possui maior tempo para atividades de lazer em decorrência da aposentadoria (OLIVEIRA et al., 2015).

Tabela 2. Prevalência de prática de atividade física no deslocamento e no lazer dos idosos. Rio do Sul, SC, 2014.

\begin{tabular}{lcc}
\multicolumn{1}{c}{ Prática de atividades físicas } & $\mathrm{f}$ & $\%{ }^{*}$ \\
\hline Atividade física no deslocamento & & 19,2 \\
$\quad$ Inativo & 41 & 50,7 \\
Insuficientemente ativo & 64 & 30 \\
$\quad$ Fisicamente ativo & 46 & 21,6 \\
Atividade física no lazer & 78 & 36,6 \\
$\quad$ Inativo & 89 & 41,8 \\
$\quad$ Insuficientemente ativo & 213 & 100 \\
Fisicamente ativo & &
\end{tabular}


Marisa Biesdorf, Jean Carlos de Souza,

Luana da Cruz Santos, Cybelle D. Soares Schlemper e

Clair Antônio Wathier

A Tabela 3 apresenta a comparação da percepção do ambiente e a prática de atividade física no deslocamento, com diferença significativa entre os idosos que relataram maior densidade residencial próxima de sua residência $(p=0,027)$, a existência de lugares para caminhar e andar de bicicleta $(p=0,032)$, melhor estética do bairro $(p=0,029)$ e maior segurança no trânsito $(p=0,005)$.

Quanto à percepção do ambiente e a prática de atividade física no deslocamento, observou-se diferença significativa entre os idosos que relataram maior densidade residencial próxima de sua residência. Conforme Hino; Reis e Florindo (2010) os locais de maior densidade populacional podem favorecer o deslocamento a pé. A maior densidade residencial pode influenciar positivamente, pois faz com que o comércio e os serviços fiquem mais próximos das residências, favorecendo o deslocamento do idoso, seja caminhando ou com uso de bicicleta.

Nesse sentido, a existência de lugar para caminhar e andar de bicicleta, somado a maior segurança no trânsito mostrou-se relacionado à prática de atividade física no deslocamento. Durante as entrevistas, percebeu-se que a falta de lugares seguros para caminhar e andar de bicicleta acaba desencorajando os idosos do município a executar suas tarefas diárias por meio da caminhada ou com o uso da bicicleta.

Outro aspecto do ambiente relacionado a atividade física de deslocamento refere-se a estética do bairro. Observou-se durante a pesquisa que ruas mal sinalizadas, esburacadas ou até mesmo sem calçadas causam restrições no deslocamento do idoso, fazendo com que desista da caminhada. Resultados semelhantes foram encontrados num estudo de revisão realizado por Owen (et al. 2004) em que ambientes com melhor estética e segurança no trânsito foram considerados como facilitadores para a prática da caminhada como forma de deslocamento. 
Giovane Pereira Balbé, O Contexto do Ambiente Percebido na Atividade Física de Lazer...

Marisa Biesdorf, Jean Carlos de Souza,

Luana da Cruz Santos, Cybelle D. Soares Schlemper e

Clair Antônio Wathier

Corroborando com estes achados, estudo realizado em São Paulo no distrito de Ermelino Matarazzo (FLORINDO et al., 2011), no ano de 2007 com 890 pessoas, com mais de 18 anos, mostrou que pessoas com percepção positiva de segurança e segurança no trânsito apresentaram mais chances de serem ativas no deslocamento. Essa percepção foi representada pelo escore geral composto pelos itens segurança para caminhar, andar de bicicleta ou praticar atividade física durante o dia ou durante a noite. Desse modo, a boa percepção de segurança dos idosos resistentes em Rio do Sul pode estar relacionada com a melhora e manutenção das estruturas dos bairros, o que proporciona uma melhor percepção da estética e da segurança do bairro. Para Wood et al. (2008) a manutenção adequada dos bairros possibilita o aumento de redes sociais e melhor controle social, contribuindo para maior sensação de segurança dos moradores.

Tabela 3. Comparação da percepção do ambiente e prática de atividade física no deslocamento dos idosos. Rio do Sul, SC, 2014.

\begin{tabular}{|c|c|c|c|c|}
\hline \multirow{3}{*}{$\begin{array}{l}\text { Domínios da percepção do } \\
\text { ambiente }\end{array}$} & \multicolumn{3}{|c|}{ Deslocamento } & \multirow{3}{*}{$\begin{array}{c}\text { Nível de } \\
\text { significância } \\
p^{*}\end{array}$} \\
\hline & Inativo & Insuficientemente ativo & Ativo & \\
\hline & & Posto Médio & & \\
\hline \multirow{2}{*}{$\begin{array}{l}\text { Densidade residencial } \\
\text { Proximidades de lojas e } \\
\text { comércio }\end{array}$} & 94,4 & 101,8 & 123,8 & \multirow{2}{*}{$\begin{array}{l}0,027 \\
0,547\end{array}$} \\
\hline & 97,7 & 108,2 & 110,8 & \\
\hline Acesso a serviços & 112,2 & 103,07 & 110,29 & \multirow{3}{*}{$\begin{array}{l}0,630 \\
0,600 \\
0,032\end{array}$} \\
\hline Conectividade das ruas & 101,61 & 105,54 & 112,92 & \\
\hline $\begin{array}{l}\text { Lugares para caminhar e } \\
\text { andar de bicicleta }\end{array}$ & 125,6 & 97,4 & 111,1 & \\
\hline Estética do bairro & 97,5 & 117,9 & 94,5 & 0,029 \\
\hline Segurança no trânsito & 78,7 & 113,8 & 113,4 & 0,005 \\
\hline Criminalidade no bairro & 116,2 & 102,7 & 108,3 & 0,473 \\
\hline
\end{tabular}

Na comparação da percepção do ambiente e prática de atividade no lazer dos idosos não houve diferenças significativas (TABELA 4). Estudo realizado em 2007, com 385 idosos, residentes no distrito de Ermelino Matarazzo em São Paulo (SALVADOR et al., 2009) concluiu que idosos com boa percepção de segurança 
Marisa Biesdorf, Jean Carlos de Souza,

Luana da Cruz Santos, Cybelle D. Soares Schlemper e

Clair Antônio Wathier

durante o dia apresentaram quatro vezes mais chances de serem ativos no lazer. Para

Hino; Reis e Florindo (2010) isso ocorre pela existência de locais mais belos, agradáveis e seguros favorecendo a utilização de espaços para a atividade física.

O modelo ecológico proposto por Sallis et al. (2006) mostrou que a prática de atividade física envolve um comportamento complexo e que mudanças nessa conduta depende das características do ambiente, mas também de outros fatores como alimentação e do próprio indivíduo. Esse modelo sugere níveis de influência e de interação envolvendo aspectos individuais, percepção sobre o ambiente e aspectos mais abrangentes, como as estruturas dos bairros e as políticas públicas.

Sendo assim, acredita-se que o acesso adequado às áreas de lazer e a espaços públicos abertos não está associado somente com a prática de atividade física no lazer ou com exercícios físicos, mas também aumenta as chances de atingir 150 minutos (semanais) de caminhada como forma de deslocamento (FOSTER; HILLSDON; THOROGOOD, 2004).

Outra hipótese para a não relação entre atividade física de lazer e os domínios do ambiente pode estar no nível socioeconômico do bairro onde os idosos residem. Estudo transversal testou a associação de atributos ambientais, avaliados objetivamente (densidade residencial, conectividade das ruas, uso misto do solo e diversidade de áreas) e subjetivamente (acesso aos destinos, estética do bairro, locais para caminhar, o tráfego/barreiras, segurança e criminalidade) com 1500 indivíduos com 57 anos ou mais de idade. Demonstrou que moradores de áreas com menor nível socioeconômico apresentaram-se menos ativos fisicamente durante o tempo de lazer, já que áreas com menor nível socioeconômico foram desfavoráveis para atividade física na relação com a densidade residencial, estética do bairro, infraestrutura para caminhar, de 
Giovane Pereira Balbé, O Contexto do Ambiente Percebido na Atividade Física de Lazer...

Marisa Biesdorf, Jean Carlos de Souza,

Luana da Cruz Santos, Cybelle D. Soares Schlemper e

Clair Antônio Wathier

tráfego/barreiras, segurança e criminalidade (SUGIYAMA et al., 2015). Este estudo sugere que a estrutura do bairro de baixo nível socioeconômico pode influenciar a atividade física de lazer da população, sendo necessário considerá-la em estudos relacionados à temática.

Tabela 4. Comparação da percepção do ambiente e prática de atividade física no lazer dos idosos. Rio do Sul, SC, 2014.

\begin{tabular}{lcccc}
\hline \multirow{2}{*}{$\begin{array}{c}\text { Domínios da percepção } \\
\text { do ambiente }\end{array}$} & Inativo & $\begin{array}{c}\text { Lazer } \\
\text { Insuficientemente ativo } \\
\text { Posto Médio }\end{array}$ & $\begin{array}{c}\text { Ativo } \\
\text { Nível de } \\
\text { significância } \\
p^{*}\end{array}$ \\
\hline $\begin{array}{l}\text { Densidade residencial } \\
\text { Proximidades de lojas e } \\
\text { comércio }\end{array}$ & 102,3 & 106,3 & 0,787 \\
Acesso a serviços & 101,5 & 105,2 & 109,9 & 0,643 \\
Conectividade das ruas & 102,4 & 105 & 11,4 & 0,691 \\
Lugares para caminhar e & 113,1 & 102,7 & 107,5 & 0,643 \\
andar de bicicleta & 110,7 & 106,4 & 105,5 & 0,887 \\
$\begin{array}{l}\text { Estética do bairro } \\
\text { Segurança no trânsito }\end{array}$ & 94,6 & 103 & 116,8 & 0,103 \\
Criminalidade no bairro & 97,2 & 110,4 & 109 & 0,471 \\
\hline$*$ Teste Kruskal-Wallis & 114,3 & 106,1 & 103,9 & 0,641 \\
\hline
\end{tabular}

* Teste Kruskal-Wallis.

\section{Conclusão}

Constatou-se uma prevalência de atividade física no lazer e no deslocamento acima do encontrado na literatura. A prática de atividade física no deslocamento foi relacionada a maior densidade residencial, locais para caminhar e andar de bicicleta, melhor estética do bairro e segurança no trânsito. Não houve relação entre a prática de atividade física de lazer e os domínios do ambiente.

Acredita-se que de posse dessas informações seja possível subsidiar políticas de planejamento urbano e de saúde pública que incluam a elaboração de intervenções para a construção e manutenção de espaços públicos favoráveis à promoção de saúde.

Dentre as limitações do estudo, pode-se citar sua natureza transversal o que não permite identificar causalidade entre os fenômenos investigados. Sugere-se que novos 
Marisa Biesdorf, Jean Carlos de Souza,

Luana da Cruz Santos, Cybelle D. Soares Schlemper e

Clair Antônio Wathier

estudos adotem medidas objetivas do ambiente e da atividade física, bem como, que passem a investigar a renda da vizinhança dos bairros em que os idosos residem e sua possível relação no nível de atividade física de lazer dessa população.

\section{REFERÊNCIAS}

BALBÉ, G. P.; WATHIER, C. A.; RECH, C. R. Características do ambiente do bairro e prática de caminhada no lazer e deslocamento em idosos. Revista Brasileira de Atividade Física e Saúde, Pelotas, v. 22, n. 2, no prelo, 2017.

BENEDETTI, T. R. B.; MAZO, G. Z.; BARROS, M. V. G. Aplicação do Questionário Internacional de Atividades Físicas para avaliação do nível de atividades físicas de mulheres idosas: validade concorrente e reprodutibilidade teste-reteste. Revista Brasileira de Ciência e Movimento, Brasília, v. 12, n. 1, p. 25-34, 2004.

BENGOECHEA, E. G.; SPENCE, J. C.; McGANNON, K. R. Gender differences in perceived environmental correlates of physical activity. International Journal Behavioral Nutrition and Physical Activity, Alberta, v. 2, p. 12, 2005.

BOOTH, M. L.; OWEN, N.; BAUMAN, A.; CLAVISI, O.; LESLIE, E. SocialCognitive and Perceived Environment Influences Associated with Physical Activity in Older Australians. Preventive Medicine, Washington, v. 31, n. 1, p. 15-22, 2000.

DUMITH, S. C.; HALlAL, P. C.; REIS, R. S.; KOHL III, H. W. Worldwide prevalence of physical inactivity and its association with human development index in 76 countries. Preventive Medicine, Washington, v. 53, p. 24-28, 2011.

FLORINDO, A. A.; SALVADOR, E. P.; REIS, R. S.; GUIMARÃES, V. V. Percepção do ambiente e prática de atividade física em adultos residentes em região de baixo nível socioeconômico. Revista de Saúde Pública, São Paulo, v. 45, n. 2, p. 302-310, 2011.

FOSTER, C.; HILLSDON, M.; THOROGOOD, M. Environmental perceptions and walking in English adults. Journal Epidemiology \& Community Health, London, v. 58, n. 11, p. 924-928, 2004.

GALlAGHER, N. A.; GRETEBECK, K. A.; ROBINSON, J. C.; TORRES, E. R.; MURPHY, S. L.; MARTYN, K. K. Neighborhood factors relevant for walking in older, urban, African American adults. Journal of Ageing and Physical Activity, Champaign, v. 18, n. 1, p. 99-115, 2010.

GIEHL, M. W. C.; SCHNEIDER, I. J. C.; CORSEUIL, H. X.; BENEDETTI, T. R. B.; d'ORSI, E. Atividade física e percepção do ambiente em idosos: estudo populacional em Florianópolis. Revista de Saúde Pública, São Paulo, v. 46, n. 3, p. 516-525, 2012. 
Marisa Biesdorf, Jean Carlos de Souza,

Luana da Cruz Santos, Cybelle D. Soares Schlemper e

Clair Antônio Wathier

HEATH, G. W; PARRA, D.; SARMIENTO, O. L.; BO ANDERSEN, L.; OWEN, N.; GOENKA, S. et al. Evidence-based intervention in physical activity: lessons from around the world. Lancet, Londres, v. 380, p. 272-281, 2012.

HENSON, J.; YATES, T.; EDWARDSON, C. L.; KHUNTI, K.; TALBOT, D.; GRAY, L. J. et al. Sedentary Time and Markers of Chronic Low-Grade Inflammation in a High Risk Population. PLoS ONE, São Francisco, v. 8, n. 10, p. 1-6, 2013.

HINO, A. A. F.; REIS, R. S.; FLORINDO, A. A. Ambiente construído e atividade física: uma breve revisão dos métodos de avaliação. Revista Brasileira de Cineantropometria \& Desempenho Humano, Florianopólis, v. 12, n. 5, p. 387-94, 2010.

HOEHNER, C. M.; RAMIREZ, L. B.; ELLIOTT, M. B.; HANDY, S. L.; BROWNSON, R. C. Perceived and objective environmental measures and physical activity among urban adults. American Journal of Preventive Medicine, Washington, v. 28, n. 2, Supl. 2, p. 105-116, 2005.

IBGE - Instituto Brasileiro de Geografia e Estatística. Censo demográfico: 2010. Disponível em: <www.cidades.ibge.gov.br>. Acesso em: 26 fev. 2014.

LIBRETT, J. J.; YORE, M. M.; SCHMID, T. L. Characteristics of physical activity levels among trail users in a U.S. national sample. American Journal of Preventive Medicine, Washington, v. 31, n. 5, p. 399-405, 2006.

MALAVASI, L. M.; DUARTE, M. F. S.; BOTH, J.; REIS, R. S. Escala de Mobilidade Ativa no Ambiente Comunitário News Brasil: tradução e reprodutibilidade. Revista Brasileira de Cineantropometria \& Desempenho Humano, Florianópolis, v. 9, n. 4, p. 339-350, 2007.

NELSON, M. E.; REJESKI, J. W.; BLAIR, S. N.; DUNCAN, P. W.; JUDGE, J. O.; KING, C. A. et al. Physical Activity and Public Health in Older Adults: Recommendation from the American College of Sports Medicine and the American Heart Association. Medicine \& Science in Sports \& Exercise, Indianapolis, v. 39, n. 8, p. 1435-1445, 2007.

OlIVEIRA, F. A; PIRAJÁ, W. C.; SIlVA, A. P.; PRIMO, C. P. F. Benefícios da prática de atividade física sistematizada no lazer de idosos: algumas considerações. Licere, Belo Horizonte, v.18, n.2, p. 262-304, 2015.

OWEN, N.; HUMPEL, N.; LESLIE, E.; BAUMAN, A.; SALLIS, J. F. Understanding environmental influences on walking; review and research agenda. American Journal of Preventive Medicine, Washington, v. 27, n. 1, p. 67-76, 2004.

PIZANI, J.; PRATES, M. E. F.; STAREPRAVO, F. A.; BARBOSA-RINALDI, I. P. O parque do Ingá como espaço público de lazer: uma análise da percepção do ambiente. Licere, Belo Horizonte, v. 18, n. 3, p. 61-85, 2015. 
Marisa Biesdorf, Jean Carlos de Souza,

Luana da Cruz Santos, Cybelle D. Soares Schlemper e

Clair Antônio Wathier

PNUD - PROGRAMA DAS NAÇÕES UNIDAS PARA O DESENVOLVIMENTO. Atlas do desenvolvimento humano no Brasil, 2013. Disponível em: http://www.pnud.org.br/atlas/ Acesso em: 27 fev. 2014.

SAELENS, B. E.; SALLIS, J. F.; BLACK, J.; CHEN, D. Measuring perceived neighborhood environment factors related to walking/cycling. Annals of Behavioral Medicine, Milwaukee, v. 24, n. S139, 2002.

SALLIS, J. F.; CERVERO, R. B.; ASCHER, W.; HENDERSON, K. A.; KRAFT, K. M.; KERR, J. An ecological approach to creating active living communities. Annual Review of Public Health, Palo Alto, v. 27, p. 297-322, 2006.

SALVADOR, E. P.; REIS, R. S.; FLORINDO, A. A. A prática de caminhada como forma de deslocamento e sua associação com a percepção do ambiente em idosos. Revista Brasileira de Atividade Física \& Saúde, Pelotas, v. 14, n. 3, p. 197-205, 2009.

; FLORINDO, A. A.; REIS, R. S.; COSTA, E. F. Percepção do ambiente e prática de atividade física no lazer entre idosos. Revista de Saúde Pública, São Paulo, v. 43, n. 6, p. 972-980, 2009.

SUGIYAMA, T.; HOWARD, N. J.; PAQUET, C.; COFFEE, N. T.; TAYLOR, A. W.; DANIEL, M. Do relationships between environmental attributes and recreational walking vary according to area-level socioeconomic status? Journal of Urban Health, Nova York, v. 21, 2015.

WOOD, L.; SHANNON, T.; BULSARA, M.; PIKORA, T.; McCORMACK, G.; GILES-CORTI, B. The anatomy of the safe and social suburb: an exploratory study of the built environment, social capital and residents' perceptions of safety. Health Place, Edimburgo, v. 14, n. 1, p. 15-31, 2008.

WHO - World Health Organization. Global recommendations on physical activity for health, Geneva: World Health Organization, 2010.

\section{Endereço dos Autores:}

Giovane Pereira Balbé

Rua Dr. Guilherme Gemballa, nº13, Jardim América

Rio do Sul - SC - 89.160-932

Endereço eletrônico: gbalbe@unidavi.edu.br

Marisa Biesdorf

Rua Dr. Guilherme Gemballa, nº13, Jardim América

Rio do Sul - SC - 89.160-932

Endereço eletrônico: marisabiesdorf@unidavi.edu.br 
Giovane Pereira Balbé,

Marisa Biesdorf, Jean Carlos de Souza,

Luana da Cruz Santos, Cybelle D. Soares Schlemper e

Clair Antônio Wathier

Jean Carlos de Souza

Rua Dr. Guilherme Gemballa, n ${ }^{\circ}$, Jardim América

Rio do Sul - SC - 89.160-932

Endereço eletrônico: jeanbittencourt@unidavi.edu.br

Luana da Cruz Santos

Rua Dr. Guilherme Gemballa, nº13, Jardim América

Rio do Sul - SC - 89.160-932

Endereço eletrônico: luana_saantos@yahoo.com.br

Cybelle Doederlein Soares Schlemper

Rua Dr. Guilherme Gemballa, ${ }^{\circ} 13$, Jardim América

Rio do Sul - SC - 89.160-932

Endereço eletrônico: cybelle_soares@yahoo.com.br

Clair Antônio Wathier

Rua Dr. Guilherme Gemballa, nº13, Jardim América

Rio do Sul - SC - 89.160-932

Endereço eletrônico: clair@unidavi.edu.br 